\title{
Linear Time Membership in a Class of Regular Expressions with Interleaving and Counting
}

\author{
Giorgio Ghelli \\ Dipartimento di Informatica - \\ Università di Pisa \\ ghelli@di.unipi.it
}

\author{
Dario Colazzo \\ LRI - Université Paris Sud \\ dario.colazzo@Iri.fr
}

\author{
Carlo Sartiani \\ Dipartimento di Informatica - \\ Università di Pisa \\ sartiani@di.unipi.it
}

\begin{abstract}
The extension of Regular Expressions (REs) with an interleaving (shuffle) operator has been proposed in many occasions, since it would be crucial to deal with unordered data. However, interleaving badly affects the complexity of basic operations, and, expecially, makes membership NPhard [13], which is unacceptable for most uses of REs.

REs form the basis of most XML type languages, such as DTDs and XML Schema types, and XDuce types [16, 11]. In this context, the interleaving operator would be a natural addition to the language of REs, as witnessed by the presence of limited forms of interleaving in XSD (the all group), Relax-NG, and SGML, provided that the NPhardness of membership could be avoided.

We present here a restricted class of REs with interleaving and counting which admits a linear membership algorithm, and which is expressive enough to cover the vast majority of real-world XML types.

We first present an algorithm for membership of a list of words into a RE with interleaving and counting, based on the translation of the RE into a set of constraints. We generalize the approach in order to check membership of XML trees into a class of EDTDs with interleaving and counting, which models the crucial aspects of DTDs and XSD schemas.
\end{abstract}

\section{Categories and Subject Descriptors}

H.2 [Database Management]: Miscellaneous

\section{General Terms}

Theory

\section{INTRODUCTION}

Given a family $L$ of extended, or restricted, regular expressions, membership for $L$ is the problem of determining whether a word $w$ belongs to the language generated by an expression $e$ in $L$. The problem is polynomial (in $|w|+|e|$ ) when $L$ is the set of standard regular expressions based on

Permission to make digital or hard copies of all or part of this work for personal or classroom use is granted without fee provided that copies are not made or distributed for profit or commercial advantage and that copies bear this notice and the full citation on the first page. To copy otherwise, to republish, to post on servers or to redistribute to lists, requires prior specific permission and/or a fee.

CIKM'08, October 26-30, 2008, Napa Valley, California, USA.

Copyright 2008 ACM 978-1-59593-991-3/08/10 ...\$5.00. union, concatenation, and Kleene star, which we denote as $R E\{+, \cdot, *\}[12]$, and is still polynomial when intersection $\cap$ is added to the operators. However, membership is NPcomplete for $R E\{+, \cdot, *, \&\}$, the set of REs extended with an interleaving operator \& (to be defined later) [13]. The simpler combinations $R E\{, \&\}, R E\{+, \&\}$ and $R E\{*, \&\}$ are already NP-hard [13], showing that the NP-hardness of membership with interleaving is quite robust.

We present here a restricted set of regular expressions with interleaving which admits a linear-time membership algorithm (using the RAM model to assess time complexity). The set contains those regular expressions where no symbol appears twice (called conflict-free or single-occurrence) and where Kleene star is only applied to symbol disjunctions. We generalize Kleene star to counting constraints such as $a[2 . .5]$ or $a[1 . . *]$. We show how this approach can be generalized to check membership of XML trees into a class of EDTDs with interleaving and counting, obtaining an algorithm whose time complexity is linear in the product of the input size with the maximal alternation depth of all the content models in the schema. In this context, the restriction we are proposing is known to be met by the vast majority of schemas that are produced in practice [10].

Our approach is based on the translation of each regular expression into a set of constraints, as in [10]. We define a linear-time translation algorithm, and we then define a linear-time algorithm to check that a word satisfies the resulting constraints. The algorithm is based on the implicit representation of the constraints using a tree structure, and on the parallel verification of all constraints, using a residuation technique. The residuation technique transforms each constraint into the constraint that has to be verified on the rest of the word after a symbol has been read; this residual constraint is computed in constant time. The notion of residuation is strongly reminiscent of the Brzozowski derivative of REs [7].

\section{TYPE AND CONSTRAINT LANGUAGE}

\subsection{The Type Language}

We follow the terminology of [10], and we use the term type instead of regular expression. We consider the following type language with counting, disjunction, concatenation, and interleaving, for strings over a finite alphabet $\Sigma$; we use $\epsilon$ for the empty word and for the expression whose language is $\{\epsilon\}$, while $a[m . . n]$, with $a \in \Sigma$, contains the words composed by $j$ repetitions of $a$, with $m \leq j \leq n$. 


$$
T::=\epsilon|a[m . . n]| T+T|T \cdot T| T \& T
$$

More precisely, we define $\mathbb{N}_{*}=\mathbb{N} \cup\{*\}$, and extend the standard order among naturals with $n \leq *$ for each $n \in \mathbb{N}_{*}$. In every type expression $a[m . . n]$ we have that $m \in(\mathbb{N} \backslash$ $\{0\}), n \in\left(\mathbb{N}_{*} \backslash\{0\}\right)$, and $n \geq m$. Specifically, $a[0 . . n]$ is not part of the language, but we use it to abbreviate $(\epsilon+$ $a[1 . . n])$. The operator $a[m . . n]$ generalizes Kleene star, but can only be applied to symbols. Unbounded repetition of a disjunction of symbols, i.e. $\left(a_{1}+\ldots+a_{n}\right) *$, can be expressed as $\left(\left(a_{1}[0 . . *]\right) \& \ldots \&\left(a_{n}[0 . . *]\right)\right)$.

String concatenation $w_{1} \cdot w_{2}$ and language concatenation $L_{1} \cdot L_{2}$ are standard. The shuffle, or interleaving, operator $w_{1} \& w_{2}$ is also standard, as follows.

Definition 2.1 ( $\left.v \& w, L_{1} \& L_{2}\right)$ The shuffle set of two words $v, w \in \Sigma^{*}$, or two languages $L_{1}, L_{2} \subseteq \Sigma^{*}$, is defined as follows; notice that each $v_{i}$ or $w_{i}$ may be the empty string $\epsilon$.

$$
\begin{gathered}
v \& w \quad={ }_{\text {def }} \quad \begin{array}{c}
\left\{v_{1} \cdot w_{1} \cdot \ldots \cdot v_{n} \cdot w_{n}\right. \\
\mid \quad v_{1} \cdot \ldots \cdot v_{n}=v, w_{1} \cdot \ldots \cdot w_{n}=w, \\
\left.v_{i} \in \Sigma^{*}, w_{i} \in \Sigma^{*}, n>0\right\}
\end{array} \\
L_{1} \& L_{2}={ }_{\text {def }} \quad \bigcup_{w_{1} \in L_{1}, w_{2} \in L_{2}} w_{1} \& w_{2}
\end{gathered}
$$

Example 2.2 $(a b) \&(X Y)$ contains the permutations of $a b X Y$ where $a$ comes before $b$ and $X$ comes before $Y$ :

$$
(a b) \&(X Y)=\{a b X Y, a X b Y, a X Y b, X a b Y, X a Y b, X Y a b\}
$$

Definition $2.3(S(w), S(T)$, Atoms $(T))$ For any string $w$, $S(w)$ is the set of all symbols appearing in $w$. For any type $T, \operatorname{Atoms}(T)$ is the set of all atoms a $[m . . n]$ appearing in $T$, and $S(T)$ is the set of all symbols appearing in $T$.

Semantics of types is defined as follows.

$$
\begin{aligned}
\llbracket \epsilon \rrbracket & =\{\epsilon\} \\
\llbracket a[m . . n] \rrbracket & =\{w|S(w)=\{a\},| w|\geq m,| w \mid \leq n\} \\
\llbracket T_{1}+T_{2} \rrbracket & =\llbracket T_{1} \rrbracket \cup \llbracket T_{2} \rrbracket \\
\llbracket T_{1} \cdot T_{2} \rrbracket & =\llbracket T_{1} \rrbracket \cdot \llbracket T_{2} \rrbracket \\
\llbracket T_{1} \& T_{2} \rrbracket & =\llbracket T_{1} \rrbracket \& \llbracket T_{2} \rrbracket
\end{aligned}
$$

We will use $\otimes$ to range over $\cdot$ and $\&$ when we need to specify common properties, such as: $\llbracket T \otimes \epsilon \rrbracket=\llbracket \epsilon \otimes T \rrbracket=\llbracket T \rrbracket$.

In this system, no type is empty. Some types contain the empty string $\epsilon$ (are nullable), and are characterized as follows.

Definition $2.4(\mathrm{~N}(T)) N(T)$ is a predicate on types, defined as follows:

$$
\begin{aligned}
N(\epsilon) & =\text { true } \\
N(a[m . . n]) & =\text { false } \\
N\left(T+T^{\prime}\right) & =N(T) \text { or } N\left(T^{\prime}\right) \\
N\left(T \otimes T^{\prime}\right) & =N(T) \text { and } N\left(T^{\prime}\right)
\end{aligned}
$$

Lemma $2.5 \epsilon \in \llbracket T \rrbracket$ iff $N(T)$.

We can now define the notion of conflict-free types.

Definition 2.6 (Conflict-free types) A type $T$ is conflictfree if for each subexpression $(U+V)$ or $(U \otimes V): S(U) \cap$ $S(V)=\emptyset$.
Equivalently, a type $T$ is conflict-free if, for any two distinct subterms $a[m . . n]$ and $a^{\prime}\left[m^{\prime} . . n^{\prime}\right]$ that occur in $T, a$ is different from $a^{\prime}$.

Remark 2.7 The class of grammars we study is quite restrictive, because of the conflict-free limitation and of the constraint on Kleene-star. However, similar, or stronger, constraints, have been widely studied in the context of DTDs and XSD schemas, and it has been discovered that the vast majority of real-life expressions do respect them.

Conflict-free REs have been studied, for example, as "duplicate-free" DTDs in [17, 14], as "Single Occurrence REs" (SOREs) in [5, 6], as "conflict-free DTDs" in [3, 2]. The specific limitation that we impose on Kleene-star is reminiscent of Chain REs (CHAREs), as defined in [5], which are slightly more restrictive. That paper states that "an examination of the 819 DTDs and XSDs gathered from the Cover Pages (including many high-quality XML standards) as well as from the web at large, reveals that more than $99 \%$ of the REs occurring in practical schemas are CHAREs (and therefore also SOREs)". Barbosa et al., on the basis of a corpus of 26604 content models from xml .org, measure that $97,7 \%$ are conflict-free, and $94 \%$ are conflict-free and simple, where simple is a restriction much stronger than our Kleene-star restriction [2]. Similar results about the prevalence of simple content models had been reported in [8].

Hereafter, we will silently assume that every type is conflictfree, although some of the properties we specify are valid for any type.

We show now how the semantics of a type $T$ can be expressed by a set of constraints. This alternative characterization of type semantics will then be used for membership checking.

\subsection{The Constraint Language}

Constraints are expressed using the following logic, where $a, b \in \Sigma$ and $A, B \subseteq \Sigma, m \in(\mathbb{N} \backslash\{0\}), n \in\left(\mathbb{N}_{*} \backslash\{0\}\right)$, and $n \geq m$ :

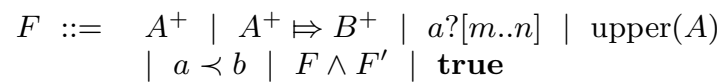

Satisfaction of a constraint $F$ by a word $w$, written $w \models F$, is defined as follows.

$$
\begin{aligned}
& w \models A^{+} \quad \Leftrightarrow \quad(S(w) \cap A) \neq \emptyset \text {, i.e. some } a \in A \\
& \text { appears in } w \\
& w \models A^{+} \Leftrightarrow B^{+} \Leftrightarrow w \not \models A^{+} \text {or } w \models B^{+} \\
& w \models a ?[m . . n] \quad \Leftrightarrow \quad \text { if } a \text { appears in } w \text {, then it appears at } \\
& (n \neq *) \quad \text { least } m \text { times and at most } n \text { times } \\
& w \models a ?[m . . *] \quad \Leftrightarrow \quad \text { if } a \text { appears in } w \text {, then it appears at } \\
& \text { least } m \text { times } \\
& w \models \operatorname{upper}(A) \Leftrightarrow S(w) \subseteq A \\
& w \models a \prec b \quad \Leftrightarrow \quad \text { there is no occurrence of } a \text { in } w \text { that } \\
& \text { follows an occurrence of } b \text { in } w \\
& w \models F_{1} \wedge F_{2} \quad \Leftrightarrow \quad w \models F_{1} \text { and } w \models F_{2} \\
& w \models \text { true } \Leftrightarrow \text { always }
\end{aligned}
$$

We use the following abbreviations:

$$
\begin{aligned}
& A^{+} \Leftrightarrow B^{+} \quad={ }_{\text {def }} \quad A^{+} \Leftrightarrow B^{+} \wedge B^{+} \Leftrightarrow A^{+} \\
& a \prec \succ b \quad={ }_{\text {def }} \quad(a \prec b) \wedge(b \prec a)
\end{aligned}
$$




$$
\begin{aligned}
A \prec B & \text { def }_{\text {def }} & & \bigwedge_{a \in A, b \in B} a \prec b \\
A \prec \succ B & \text { def }_{\text {def }} & & \bigwedge_{a \in A, b \in B} a \prec \succ b \\
\text { false } & & \text { def }_{\text {def }} & \emptyset^{+} \\
A^{-} & & ={ }_{\text {def }} & A^{+} \mapsto \emptyset^{+}
\end{aligned}
$$

The next propositions specify that $A \prec \succ B$ encodes mutual exclusion between sets of symbols, and that $A^{-}$denotes the absence of any symbol in $A$.

Proposition $2.8 w \models a \prec \succ b \Leftrightarrow a$ and $b$ are not both in $S(w)$.

Proposition $2.9 w \models A \prec \succ B \Leftrightarrow w \not \models A^{+} \wedge B^{+}$

Proposition 2.10 $w \models A^{-} \Leftrightarrow w \not \models A^{+}$

\subsection{Constraint Extraction}

We can now define the extraction of constraints from types. To each type $T$, we associate a formula $S^{+}(T)$ that tests for the presence of one of its symbols; $S^{+}(T)$ is defined as $(S(T))^{+}$.

We can now endow a type $T$ with five sets of constraints, which hold for every word $w \in \llbracket T \rrbracket$. We start with those constraints whose definition is flat, since they only depend on the leaves of the syntax tree of $T$ :

- lower-bound: unless $T$ is nullable (i.e., unless $\mathrm{N}(\mathrm{T})$ ), $w$ must include one symbol of $S(T)$;

- cardinality: if a symbol in $S(T)$ appears in $w$, it must appear with the right cardinality;

- upper-bound: no symbol out of $S(T)$ may appear in $w$.

Definition 2.11 (Flat constraints)

Lower-bound:

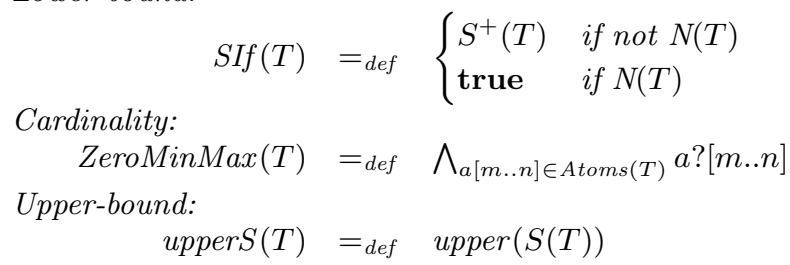

Flat constraints:

$$
\begin{aligned}
\mathcal{F C}(T)=\text { def } & S I f(T) \wedge \operatorname{ZeroMinMax}(T) \\
& \wedge \operatorname{upper} S(T)
\end{aligned}
$$

We add now the nested constraints, whose definition depends on the internal structure of $T$; the quantification "for any $w \in \llbracket C\left[T^{\prime}\right] \rrbracket$ " below means "for any $w \in T$ where $T$ is any type with a subterm $T^{\prime \prime}$. All the nested constraints depend on the fact that $T$ is conflict-free.

- co-occurrence: for any $w \in \llbracket C\left[T_{1} \otimes T_{2}\right] \rrbracket$, unless $T_{2}$ is nullable, if a symbol in $S\left(T_{1}\right)$ is in $w$, then a symbol in $S\left(T_{2}\right)$ is in $w$ as well; unless $T_{1}$ is nullable, if a symbol in $S\left(T_{2}\right)$ is in $w$, then a symbol in $S\left(T_{1}\right)$ is in $w$ as well;

- order: for any $w \in \llbracket C\left[T_{1} \cdot T_{2}\right] \rrbracket$, no symbol in $S\left(T_{1}\right)$ may follow a symbol in $S\left(T_{2}\right)$;

- exclusion: for any $w \in \llbracket C\left[T_{1}+T_{2}\right] \rrbracket$, it is not possible that $w$ has a symbol in $S\left(T_{1}\right)$ and also a symbol in $S\left(T_{2}\right)$.
In the formal definition below, $\operatorname{If}_{T_{2}}\left(S^{+}\left(T_{1}\right) \Leftrightarrow S^{+}\left(T_{2}\right)\right)$ denotes, by definition, true when $\mathrm{N}\left(T_{2}\right)$, and $\left(S\left(T_{1}\right)\right)^{+} \Leftrightarrow$ $\left(S\left(T_{2}\right)\right)^{+}$otherwise. Observe that the exclusion constraints are actually encoded as order constraints.

\section{Definition 2.12 (Nested constraints)}

Co-occurrence:

$$
\begin{array}{rll}
\mathcal{C C}\left(T_{1} \otimes T_{2}\right)={ }_{\text {def }} & \text { If }_{T_{2}}\left(S^{+}\left(T_{1}\right) \boxminus S^{+}\left(T_{2}\right)\right) \\
& & \wedge I f_{T_{1}}\left(S^{+}\left(T_{2}\right) \boxminus S^{+}\left(T_{1}\right)\right) \\
\mathcal{C C}(T)={ }_{\text {def }} & \text { true otherwise }
\end{array}
$$

Order/exclusion:

$$
\begin{array}{rll}
\mathcal{O C}\left(T_{1}+T_{2}\right) & ={ }_{\text {def }} & S\left(T_{1}\right) \prec \succ S\left(T_{2}\right) \\
\mathcal{O C}\left(T_{1} \cdot T_{2}\right) & \text { def }_{\text {def }} & S\left(T_{1}\right) \prec S\left(T_{2}\right) \\
\mathcal{O C}(T) & =\text { def }_{\text {drue }} & \text { truerwise }
\end{array}
$$

Nested constraints:

$$
\mathcal{N C}(T)={ }_{\text {def }} \bigwedge_{T_{i} \text { subterm of } T}\left(\mathcal{C C}\left(T_{i}\right) \wedge \mathcal{O C}\left(T_{i}\right)\right)
$$

As a consequence of the above definition, nested constraints have the following property.

\section{Proposition 2.13 $(\mathcal{N C}(T))$}

$$
\begin{aligned}
& \mathcal{N C}\left(T_{1}+T_{2}\right)=\left(S\left(T_{1}\right) \prec \succ S\left(T_{2}\right)\right) \wedge \mathcal{N C}\left(T_{1}\right) \wedge \mathcal{N C}\left(T_{2}\right) \\
& \mathcal{N C}\left(T_{1} \& T_{2}\right)=\quad I f_{T_{2}}\left(S^{+}\left(T_{1}\right) \models S^{+}\left(T_{2}\right)\right) \wedge \\
& \wedge I f_{T_{1}}\left(S^{+}\left(T_{2}\right) \Leftrightarrow S^{+}\left(T_{1}\right)\right) \wedge \\
& \mathcal{N C}\left(T_{1}\right) \wedge \mathcal{N C}\left(T_{2}\right) \\
& \mathcal{N C}\left(T_{1} \cdot T_{2}\right)=\quad\left(S\left(T_{1}\right) \prec S\left(T_{2}\right)\right) \\
& \wedge I f_{T_{2}}\left(S^{+}\left(T_{1}\right) \Leftrightarrow S^{+}\left(T_{2}\right)\right) \wedge \\
& \wedge I f_{T_{1}}\left(S^{+}\left(T_{2}\right) \Leftrightarrow S^{+}\left(T_{1}\right)\right) \wedge \\
& \wedge \mathcal{N} \mathcal{C}\left(T_{1}\right) \wedge \mathcal{N C}\left(T_{2}\right) \\
& \mathcal{N C}(\epsilon)=\mathcal{N C}(a[m . . n])=\text { true }
\end{aligned}
$$

By definition, when either $A$ or $B$ is " $\emptyset$ ", both $A \prec B$ and $A \prec \succ B$ are true, hence the order constraint associated to a node where one child has $S\left(T_{i}\right)=\emptyset$ is trivial; this typically happens with a subterm $T+\epsilon$. For an example of nested constraint extraction, see the upper part of Figure 1.

The following theorem is proved in [10] and states that constraints provide a sound and complete characterization of type semantics.

Theorem 2.14 Given a conflict-free type $T$, it holds that:

$$
w \in \llbracket T \rrbracket \quad \Leftrightarrow \quad w \models \mathcal{F C}(T) \wedge \mathcal{N C}(T)
$$

This theorem allows us to reduce membership in $T$ to the verification of $\mathcal{F C}(T) \wedge \mathcal{N C}(T)$.

\section{BASIC RESIDUATION ALGORITHM}

We first present an algorithm to decide membership of a word $w$ in a type $T$ in time $O(|w| * \operatorname{depth}(T))$, where $\operatorname{depth}(T)$ is defined as $\operatorname{depth}(\epsilon)=\operatorname{depth}(a[m . . n])=1, \operatorname{depth}\left(T_{1} \circledast\right.$ $\left.T_{2}\right)=1+\max \left(\operatorname{depth}\left(T_{1}\right), \operatorname{depth}\left(T_{2}\right)\right)$, where $\circledast$ stands for any binary operator. The algorithm verifies whether the word satisfies all the constraints associated with $T$, through a linear scan of $w$. The basic observation is that every symbol $a$ of $w$ transforms each constraint $F$ into a residual constraint $F^{\prime}$, to be satisfied by the subword $w^{\prime}$ that follows the symbol, according to Table 1 . We write $F \stackrel{a}{\rightarrow} F^{\prime}$ to specify that $F$ is transformed into $F^{\prime}$ by $a$; in all the cases not covered by Table 1 , we have that $F \stackrel{a}{\rightarrow} F$. We apply residuation to the nested constraints only, since flat constraints can be 
Co-occurrence
\begin{tabular}{|l|l|l|l|l|l|}
\hline Condition & $a \in A$ & $a \in B$ & $a \in A$ & $a \in B$ & $a \in A$ \\
\hline Constraint & $A^{+} \Leftrightarrow B^{+}$ & $A^{+} \Leftrightarrow B^{+}$ & $A^{+} \Leftrightarrow B^{+}$ & $A^{+} \Leftrightarrow B^{+}$ & $A^{+}$ \\
\hline Residual & $B^{+}$ & true & $B^{+}$ & $A^{+}$ & true \\
\hline
\end{tabular}

Order

\begin{tabular}{|l|l|l|l|l|l|}
\hline Condition & $a \in A$ & $a \in B$ & $a \in A$ & $a \in B$ & $a \in A$ \\
\hline Constraint & $A \prec B$ & $A \prec B$ & $A \prec \succ B$ & $A \prec \succ B$ & $A^{-}$ \\
\hline Residual & $A \prec B$ & $A^{-}$ & $B^{-}$ & $A^{-}$ & false \\
\hline
\end{tabular}

Table 1: Computing the residual of a nested constraint

checked in linear time by just counting the occurrences of each symbol in the word.

Residuation is extended from symbols to non-empty sequences of symbols in the obvious way:

$$
\begin{array}{ll} 
& F \stackrel{a}{\rightarrow} F^{\prime} \\
w \neq \epsilon: \quad & F \stackrel{a}{\rightarrow} F^{\prime} \wedge F^{\prime} \stackrel{w}{\rightarrow} F^{\prime \prime} \quad \Rightarrow \quad F \stackrel{a}{\rightarrow}+F^{\prime} \\
\stackrel{a w}{\rightarrow}+F^{\prime \prime}
\end{array}
$$

When a word has been read up to the end, the residual constraint is satisfied iff it is satisfied by $\epsilon$, that is, if it is different from $A^{+}$or false. This is formalized by the relations $F \rightarrow{ }^{\epsilon} G$ and $F \stackrel{w}{\rightarrow} *$, defined below, with $G \in$ \{true, false $\}$.

$$
\begin{aligned}
& A^{+} \Leftrightarrow B^{+} \rightarrow^{\epsilon} \text { true } \quad A^{+} \Leftrightarrow B^{+} \rightarrow^{\epsilon} \text { true } \\
& A^{+} \quad \rightarrow^{\epsilon} \text { false false } \rightarrow^{\epsilon} \text { false } \\
& A \prec B \quad \rightarrow^{\epsilon} \text { true } \quad A \prec \succ B \rightarrow^{\epsilon} \text { true } \\
& A^{-} \quad \rightarrow^{\epsilon} \text { true true } \rightarrow^{\epsilon} \text { true } \\
& F \rightarrow^{\epsilon} G \quad \Rightarrow F \stackrel{\epsilon}{\rightarrow}^{*} G \\
& F \stackrel{w}{\rightarrow}+F^{\prime} \wedge F^{\prime} \rightarrow^{\epsilon} G \Rightarrow F \stackrel{w}{\rightarrow}^{*} G
\end{aligned}
$$

The following lemma specifies that residuation corresponds to the semantics of our constraints.

Lemma 3.1 (Residuation) $w \models F$ iff $F \stackrel{w}{\rightarrow}$ true.

Residuation gives us immediately a membership algorithm of complexity $O(|w| *|\mathcal{N C}(T)|)$ : for each symbol $a$ in $w$, and for each constraint $F$ in $\mathcal{N C}(T)$, we substitute $F$ with the residual $F^{\prime}$ such that $F \stackrel{a}{\rightarrow} F^{\prime}$. The word $w$ is in $T$ iff no false or $A^{+}$is in the final set of constraints.

However, as discussed later, we can do much better than $O(|w| *|\mathcal{N C}(T)|)$. First of all, we do not build the constraints, but we keep them, and their residuals, implicit in a tree-shaped data structure with size $O(|T|)$. The structure initially corresponds to the syntax tree of $T$, encoded as a set of nodes and a Parent [] array, such that, for each node $n$, Parent $[n]$ is either null or a pair $\left(n_{p}\right.$,direction $) ; n_{p}$ is the parent of $n$, while direction is left if $n$ is the left child of $n_{p}$, and is right if it is the right child (Figure 1).

The constraints, and their residuals, are encoded using the following arrays, defined on the same nodes:

- CC[]: for each node $n$ of $T$, such that $A_{1}$ and $A_{2}$ are, respectively, the symbols in the left and right descendants of $n, \mathrm{CC}[n]$ is a symbol in the finite set $\left\{\Leftrightarrow, \Leftrightarrow, \Leftrightarrow, L^{+}, R^{+}\right.$, true $\}$that specifies the status of the associated co-occurrence constraint, as follows:

$$
\begin{aligned}
& -\Leftrightarrow \text { : encodes } A_{1}{ }^{+} \Leftrightarrow A_{2}{ }^{+} ; \\
& -\Leftrightarrow: \text { encodes } A_{1}{ }^{+} \Leftrightarrow A_{2}{ }^{+} ; \\
& -\Leftrightarrow: \text { encodes } A_{2}{ }^{+} \Leftrightarrow A_{1}{ }^{+} ;
\end{aligned}
$$
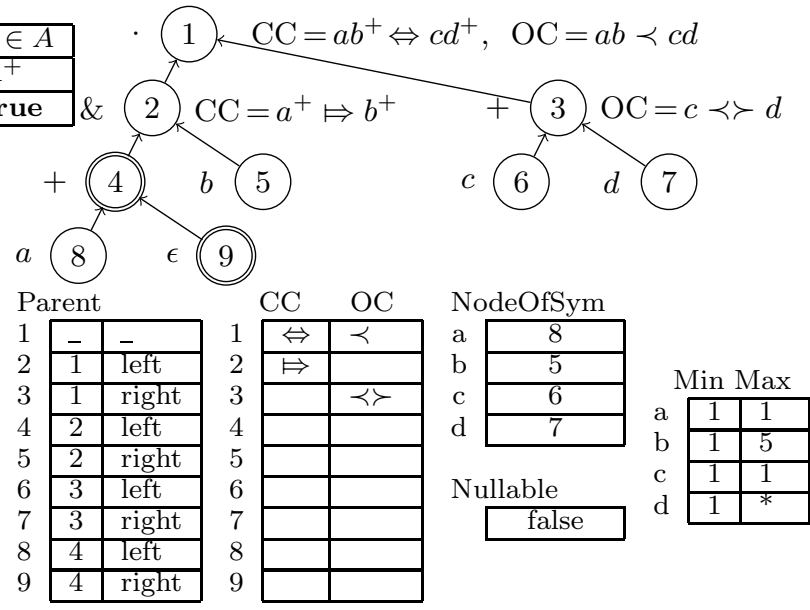

Figure 1: Syntax tree for $T=((a+\epsilon) \& b[1 . .5]) \cdot\left(c+d^{+}\right)$, and the corresponding algorithmic representation; the two nullable nodes have a double line in the picture

- $L^{+}$: encodes the residual constraint $A_{1}^{+}$;

- $R^{+}$: encodes the residual constraint $A_{2}^{+}$;

- true: encodes true.

- $\mathrm{OC}[]$ : for each node $n$, $\mathrm{OC}[n]$ specifies the status of the associated order constraint, and may assume the following values:

- $\prec$ : encodes $A_{1} \prec A_{2}$;

- $\succ$ : encodes $A_{1} \prec \succ A_{2}$;

$-L^{-}$: encodes the residual constraint $A_{1}{ }^{-}$;

$-R^{-}$: encodes the residual constraint $A_{2}{ }^{-}$;

- false: encodes the residual constraint false.

- NodeOfSymbol[]: NodeOfSymbol $[a]$ is the only node $n_{a}$ associated with a type $a[m . . n]$, for some $m$ and $n$, and is null if no such node exists ${ }^{1}$;

- $\operatorname{Min}[] / \operatorname{Max}[]: \operatorname{Min}[a]$ and $\operatorname{Max}[a]$, when different from null, encode a constraint $a ?[\operatorname{Min}[a] . . \operatorname{Max}[a]]$.

- Nullable: Nullable is not an array, but is just a boolean that is true iff $T$ is nullable.

Table 2 reports the constraint symbols that are initially associated with a node $n$ that corresponds to a subterm $T^{\prime}$ of the input type. The co-occurrence constraint depends on the nullability of $T_{1}$ and $T_{2}$.

After the constraint representation has been built, the algorithm (Figure 3) reads each character $a$ from the input word $w$, scans the ancestors of $a[m . . n]$ in the constraint tree, residuates all the constraints in this branch, and keeps track of all the $A^{+}$constraints that are so generated. At the end of $w$, it checks that all the $A^{+}$constraints have been further residuated into true. It also verifies that each symbol respects its cardinality constraints, using the Count [] array

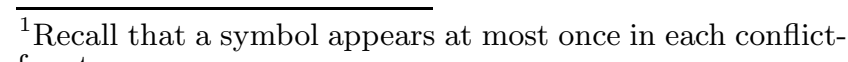
free type 


\begin{tabular}{|l|l|l|l|l|}
\hline$T^{\prime}$ & $\mathrm{N}\left(T_{1}\right)$ & $\mathrm{N}\left(T_{2}\right)$ & $C C[n]$ & $O C[n]$ \\
\hline \hline$T 1 \cdot T 2$ & true & true & true & $\prec$ \\
\hline$T 1 \cdot T 2$ & true & false & $\Leftrightarrow$ & $\prec$ \\
\hline$T 1 \cdot T 2$ & false & true & $\Leftrightarrow$ & $\prec$ \\
\hline$T 1 \cdot T 2$ & false & false & $\Leftrightarrow$ & $\prec$ \\
\hline$T 1 \& T 2$ & true & true & true & true \\
\hline$T 1 \& T 2$ & true & false & $\Leftrightarrow$ & true \\
\hline$T 1 \& T 2$ & false & true & $\Leftrightarrow$ & true \\
\hline$T 1 \& T 2$ & false & false & $\Leftrightarrow$ & true \\
\hline$T 1+T 2$ & $S\left(T_{1}\right)=\emptyset \vee S\left(T_{2}\right)=\emptyset$ & true & true \\
\hline$T 1+T 2$ & \multicolumn{2}{|l|}{$S\left(T_{1}\right) \neq \emptyset \wedge S\left(T_{2}\right) \neq \emptyset$} & true & $\prec \succ$ \\
\hline
\end{tabular}

Table 2: Initialization of constraint annotations.

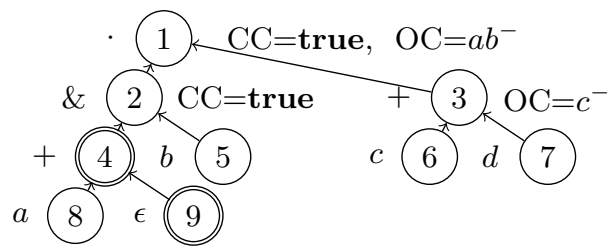

Figure 2: The tree for $T=((a+\epsilon) \& b[1 . .5]) \cdot\left(c+d^{+}\right)$ after word $b b a c$ has been read

to record the cardinalities, and the Cardinality $O K$ function to verify the constraints. This final check is clearly linear in $w$.

Example 3.2 Consider the type $T=((a+\epsilon) \& b[1 . .5]) \cdot(c+$ $\left.d^{+}\right)$, where we use $a$ to abbreviate $a[1 . .1]$ and $a^{+}$to abbreviate $a[1 . . *]$. Its syntax tree is reported in Figure 1.

Assume we read a word bbac. When $b$ is read, the value of Count $[b]$ is set to 1 . The node 5 is retrieved from NodeOfSymbol [b], and its ancestors (2,right) and (1,left) are visited. The constraint $C C[2]$ is $\Leftrightarrow$, the direction of $b$ is right, hence the constraint becomes true. The constraint $C C[1]$ is $\Leftrightarrow$, the direction is left, hence it becomes $R^{+}$. Node 1 is also pushed into ToCheck, since the algorithm must eventually check that every $R^{+}$or $L^{+}$has been residuated to true. Finally, $O C[1]$ is not affected. When the next $b$ is read, Count $[b]$ becomes 2 (we will ignore Count [] for the next letters), and its ancestors are visited again, but this time none of them is changed. When $a$ is read, $C C[2]$ is true, hence is not affected, and $C C[1]$ is $R^{+}$, hence is not affected. When $c$ is read, its ancestors (3,left) and (1, right) are visited. $O C[3]$ becomes $R^{-}$and $O C[1]$ becomes $L^{-}$, so that the tree is now the one represented in figure 2 .

The algorithm now verifies that Count [] respects the cardinality constraints and that every $A^{+}$node pushed into ToCheck has been residuated to true; since both checks succeed, it returns true. If the word had been bbacb... instead, the algorithm would now find a $b$, visit nodes (2, right) and $(1$, left $)$, and, finding a $L^{-}$in node 1 , would return false immediately.

The constraint tree can be built in time $O(|T|)$. Moreover, for every symbol $a$ in $w$, we only search and update the nodes which are ancestors of NodeOfSymbol $[a]$, and any such update has a constant cost, hence the resulting algorithm runs in $O(|T|+|w| * \operatorname{depth}(T))$.

Theorem 3.3 (complexity) $\operatorname{Member}(w, T)$ runs in time $O(|T|+|w| * \operatorname{depth}(T))$.

We can now prove that the algorithm is correct.

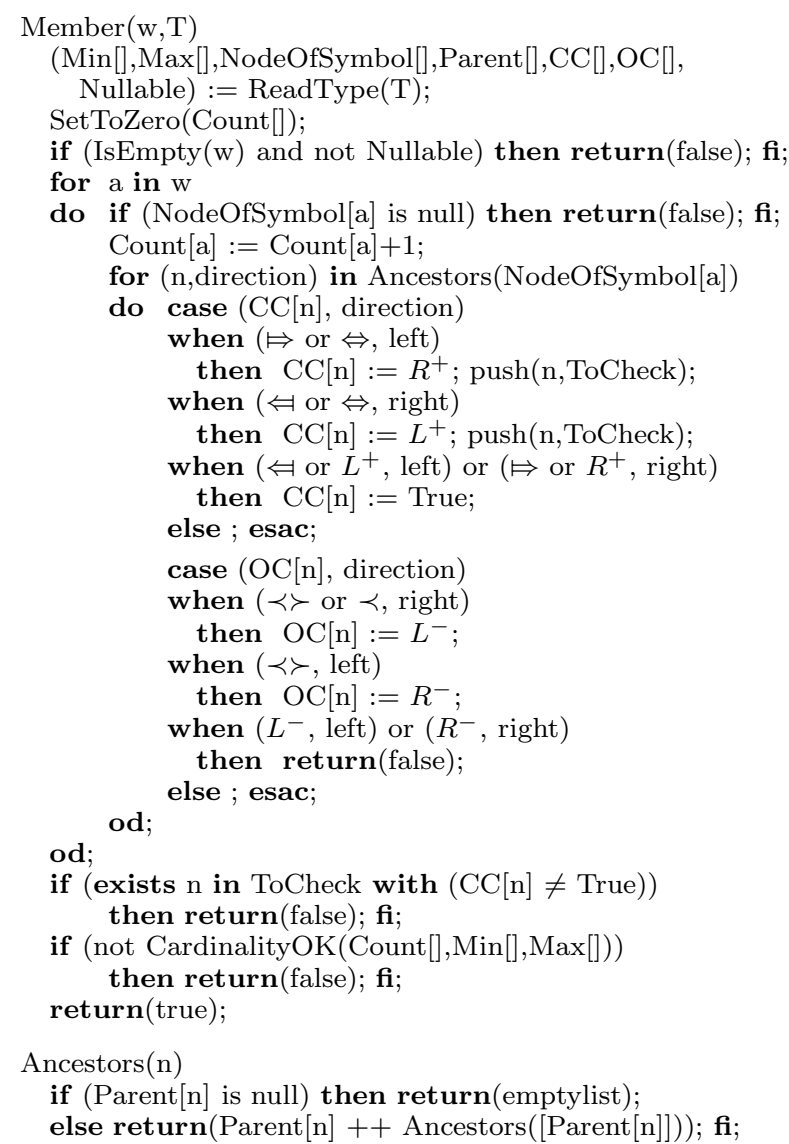

Figure 3: The basic residuation algorithm.

Theorem 3.4 (soundness) Member $(w, T)$ yields true iff $w \in \llbracket T \rrbracket$.

\section{THE "ALMOST LINEAR" VERSION}

The value of $\operatorname{depth}(T)$ can be quite large, in practice, for example for types with many fields, such as $T_{1} \ldots T_{n}$, or for types with many alternatives, such as $T_{1}+\ldots+T_{n}$; in this last case, the type may be much larger than the word itself. This problem can be easily solved by flattening the constraints generated by such types, as follows $\left(\operatorname{SIf}\left(T_{1}, \ldots, T_{n}\right)\right.$ stands for $\left\{S^{+}\left(T_{i}\right) \mid \operatorname{not} \mathrm{N}\left(T_{i}\right)\right\}$; if $\operatorname{SIf}\left(T_{1}, \ldots, T_{n}\right)=\emptyset$, then $\mathcal{C C}\left(T_{1} \otimes \ldots \otimes T_{n}\right)$ is just true). Informally, for each product type $T_{1} \otimes \ldots \otimes T_{n}$, if any symbol from any $T_{i}$ appears in $w$, then every non-nullable $T_{i}$ must contribute at least a symbol to $w$; the other cases are simple.

$$
\begin{aligned}
\mathcal{C C}\left(T_{1} \otimes \ldots \otimes T_{n}\right)= & \left(\cup_{i \in 1 \ldots n} S\left(T_{i}\right)\right)^{+} \\
& \mapsto S I f\left(T_{1}, \ldots, T_{n}\right) \\
\mathcal{O C}\left(T_{1} \cdot \ldots T_{n}\right)= & S\left(T_{1}\right) \prec \ldots \prec S\left(T_{n}\right) \\
\mathcal{O C}\left(T_{1}+\ldots+T_{n}\right)= & \prec \succ\left(S\left(T_{1}\right), \ldots, S\left(T_{n}\right)\right)
\end{aligned}
$$

Formally, we consider the three n-ary operators above in the syntax for types, and we generalize the syntax for constraints as specified below. The constraints $A_{1}{ }^{-}, \ldots, A_{i}{ }^{-}$ and $A_{1} \prec \ldots \prec A_{j}$ are just two special cases of the constraint $A_{1}{ }^{-}, \ldots, A_{i}{ }^{-}, A_{i+1} \prec \ldots \prec A_{i+j}$, when $j=0$ or $i=0$ respectively; when both are zero, the constraint is writ- 
ten "true". As usual, false abbreviates $(\emptyset)^{+}$. The syntactic forms below are defined under the condition that $i \geq 0$, $j \geq 0$, and $A_{0} \supseteq\left(A_{1} \cup \ldots \cup A_{i+1}\right)$.

$$
\begin{aligned}
F::=\quad & A_{0}{ }^{+} \mapsto\left\{A_{1}{ }^{+}, \ldots, A_{i+1}{ }^{+}\right\} \\
& \mid \begin{array}{ll}
A_{1}{ }^{+}, \ldots, A_{i+1}{ }^{+} \\
\prec \succ\left(A_{1}, \ldots, A_{i+2}\right) \\
\mid & A_{1}^{-}, \ldots, A_{i}^{-}, A_{i+1} \prec \ldots \prec A_{i+j}
\end{array}
\end{aligned}
$$

The semantics of these new constraints is fully defined by Table 3 plus the final conditions $A_{1}{ }^{+}, \ldots, A_{n}{ }^{+} \rightarrow^{\epsilon}$ false and $F \rightarrow{ }^{\epsilon}$ true otherwise.

We correspondingly refine the data structures of the residuation algorithm, as follows. Co-occurrence constraints are represented by an array $C C[]$ of records with the following fields: $C C[n]$. kind $\in\left\{\Leftrightarrow, A^{+}\right.$, true $\}, C C[n]$. needed [] , which is an array of booleans, and $C C[n]$.neededCount $\in \mathbb{N}$. Informally, if we have a type $T_{1} \otimes T_{2} \otimes T_{3}$, where $T_{1}$ is the only nullable child, we have a constraint $S^{+}\left(T_{1} \otimes T_{2} \otimes T_{3}\right) \Leftrightarrow$ $\left(S^{+}\left(T_{2}\right), S^{+}\left(T_{3}\right)\right)$, and we represent it through a record $c c$ with "cc.kind $=(\Leftrightarrow)$ ", with cc.needed []$=[$ false, true, true $]$, specifying that $S^{+}\left(T_{1}\right)$ is "not needed" (since $T_{1}$ is nullable), while $S^{+}\left(T_{2}\right)$ and $S^{+}\left(T_{3}\right)$ are "needed"; cc.neededCount would contain 2 .

The first time we meet any children $i$, we switch the kind of $c c$ from $(\Leftrightarrow)$ to $\left(A^{+}\right)$, and we set $c c . n e e d e d[i]$ to false. For any other child $i^{\prime}$ we meet, we will also set its cc.needed $\left[i^{\prime}\right]$ value to false. Every time we switch a needed [] entry from true to false, we also decrease the value of cc.neededCount, so that any constraint of kind $\left(A^{+}\right)$is satisfied when its cc.neededCount is down to zero.

Order constraints are represented by an array $O C[]$ of records with fields kind $\in\left\{-\prec, \prec \succ, A^{-}\right.$, true $\}$, and allowed $\in \mathbb{N}$. If we have a type $T_{1} \cdot \ldots \cdot T_{m}$, the corresponding $o c$ record has oc.kind $=\left({ }^{-} \prec\right)$ and oc.allowed $=1$, which corresponds to a constraint $S\left(T_{1}\right) \prec \ldots \prec S\left(T_{m}\right)$. More generally, oc.kind $=(-\prec)$ with oc.allowed $=i$, represents a constraint $A_{1}{ }^{-}, \ldots, A_{i-1}{ }^{-}, A_{i} \prec \ldots \prec A_{m}$, hence, when we meet a symbol in $S\left(T_{j}\right)$, if $j<$ allowed we return false, and otherwise we just update oc.allowed to $j$.

Finally, a type $T_{1}+\cdots+T_{m}$ is represented by $o c$ with oc.kind $=(\prec \succ)$, which is residuated into oc.kind $=\left(A^{-}\right)$and oc. allowed $=i$ when a symbol in $S\left(T_{i}\right)$ is met, and yields false if, later on, a symbol in $S\left(T_{i^{\prime}}\right)$ is met with $i^{\prime} \neq i$.

To sum up, we represent these constraints, and their residuals, through the following two arrays; we assume that $n$ is the node that corresponds to a type $T$ whose children are $T_{1}, \ldots, T_{m}$.

- CC[]: for each node $n, \mathrm{CC}[n]$ is a record with fields kind, neededCount and needed [], whose meaning depends on the value of $C C[n]$.kind, as follows:

$$
\begin{aligned}
-\mapsto: C C[n] \text { represents a constraint } \\
\\
\quad\left(\cup_{i \in 1 . . n} S\left(T_{i}\right)\right)^{+} \Leftrightarrow\left\{S\left(T_{k(1)}\right)^{+}, \ldots, S\left(T_{k(j)}\right)^{+}\right\}
\end{aligned}
$$

where $j$ is $C C[n] . n e e d e d C o u n t$, and where $\{k(1), \ldots, k(j)\}$ enumerates the indexes $i$ such that $C C[n]$.needed $[i]=$ true.

- $A^{+}: C C[n]$ represents a constraint

$$
S\left(T_{k(1)}\right)^{+}, \ldots, S\left(T_{k(j)}\right)^{+}
$$

where $j$ is $C C[n]$. neededCount, and where

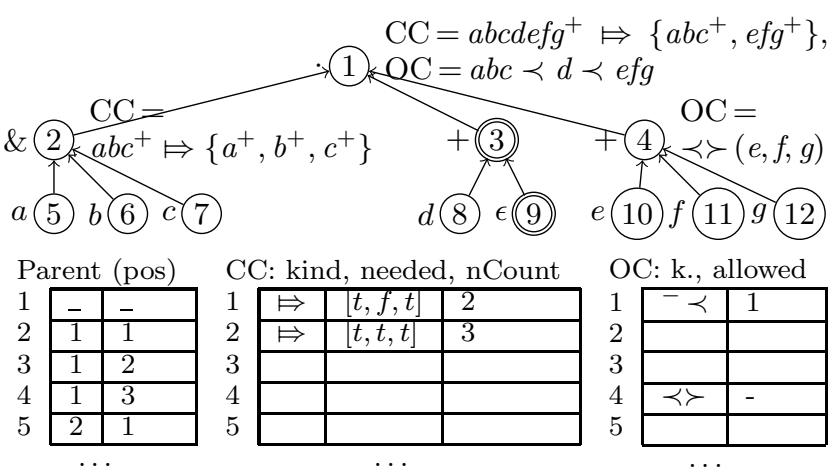

Figure 4: Representation of $T=(a \& b \& c) \cdot\left(d^{*}\right) \cdot(e+f+g)$

$\{k(1), \ldots, k(j)\}$ enumerates the indexes $i$ such that $C C[n]$. needed $[i]=$ true.

- OC[]: for each node $n$ with $m$ children, $\mathrm{CC}[n]$ is a record with two fields kind and allowed, whose meaning depends on the kind field, as follows:

${ }^{-}{ }^{-} \prec$ : this is a constraint

$$
A_{1}{ }^{-}, \ldots, A_{i-1}{ }^{-}, A_{i} \prec \ldots \prec A_{m}
$$

where $A_{j}=S\left(T_{j}\right)$, and $i=C C[n]$.allowed.

- $\succ$ : this is a constraint $A_{1}{ }^{-}, \ldots, A_{m}{ }^{-}$, where $A_{j}=S\left(T_{j}\right)$;

$-A^{-}$: this is a residual constraint

$$
A_{1}{ }^{-}, \ldots, A_{i-1}{ }^{-}, A_{i+1}{ }^{-}, \ldots, A_{m}{ }^{-}
$$

where $A_{j}=S\left(T_{j}\right)$ and $i=C C[n]$.allowed.

We present the modified parts of the algorithm in Figure 5.

Example 4.1 Consider the type $T=(a \& b \& c) \cdot\left(d^{*}\right) \cdot(e+$ $f+g$ ) (Figure 4), where we use $a$ to abbreviate $a[1 . .1]$ and $a^{*}$ to abbreviate $a[1 . . *]+\epsilon$.

The record $O C[3]$ is null since only one child has a nonempty set of symbols. Assume we read a word bcadddgdga. When $b$ is read, nodes 2 and 1 are visited, and their corresponding constraints are residuated to: $C C[2]=\left(A^{+},[t, f, t], 2\right)$, $C C[1]=\left(A^{+},[f, f, t], 1\right), O C[1]=\left({ }^{-} \prec, 1\right)$. The constraint $O C[1]$ is actually unaffected, because both allowed and the child position pos are equal to 1 . Both 2 and 1 are inserted in ToCheck, since they have now an $A^{+}$kind. When $c$ is read, $C C[2]$ becomes $\left(A^{+},[t, f, f], 1\right)$ and the constraints in node 1 are unaffected. When $a$ is read, $C C[2]$ becomes (true, $[f, f, f], 0)$, since its neededCount is 0 . When $d$ is read, 3 and 1 are visited; $C C[1]$ is unaffected, since $C C[1][2]$ is already false, while $O C[1]$ becomes $\left({ }^{-} \prec, 2\right)$, which means that symbols from the first subtree are now disallowed. The next two $d$ 's require two new visits to 3 and 1 , which have no effect. When $g$ is read, $O C[4]$ becomes $\left(A^{-}, 3\right), C C[1]$ becomes (true, $[f, f, f], 0)$, and $O C[1]$ becomes $\left({ }^{-} \prec, 3\right)$. If the word ended here, the algorithm would return true, since both nodes in ToCheck have now kind true. But now a new $d$ is read, which would residuate $O C[1]$ to false, hence the algorithm stops with false.

This version of the algorithm is in $O(|T|+|w|$ *flatdepth $(T))$, where flatdepth $(T)$ is the depth of the type after all operators have been flattened. In practice, flatdepth $(T)$ is almost 


\begin{tabular}{|l|l|l|}
\hline Condition & Constraint & Residual after $a$ \\
\hline$a \in A_{i} \subseteq A$ & $A^{+} \Leftrightarrow\left\{A_{1}{ }^{+}, \ldots, A_{n}{ }^{+}\right\}$ & $A_{1}{ }^{+}, \ldots, A_{i-1}{ }^{+}, A_{i+1}+, \ldots, A_{n}{ }^{+}$ \\
\hline \hline$a \in A$ & $A^{+}$ & true \\
\hline$a \in A_{i}, n>1$ & $A_{1}^{+}, \ldots, A_{n}{ }^{+}$ & $A_{1}^{+}, \ldots, A_{i-1}{ }^{+}, A_{i+1}{ }^{+}, \ldots, A_{n}{ }^{+}$ \\
\hline \hline$a \in A_{j}$ & $\prec \succ\left(A_{1}, \ldots, A_{n}\right)$ & $A_{1}^{-}, \ldots, A_{j-1}{ }^{-}, A_{j+1}-, \ldots, A_{n}{ }^{-}$ \\
\hline \hline$a \in A_{j}, j \leq i$ & $A_{1}^{-}, \ldots, A_{i}^{-}, A_{i+1} \prec \ldots \prec A_{n}$ & false \\
\hline$a \in A_{j}, j>i$ & $A_{1}, \ldots, A_{i}^{-}, A_{i+1} \prec \ldots \prec A_{n}$ & $A_{1}^{-}, \ldots, A_{j-1}, A_{j} \prec \ldots \prec A_{n}$ \\
\hline
\end{tabular}

Table 3: Residuals of flattened constraints

invariably smaller than three (see [4]), hence this algorithm is "almost linear".

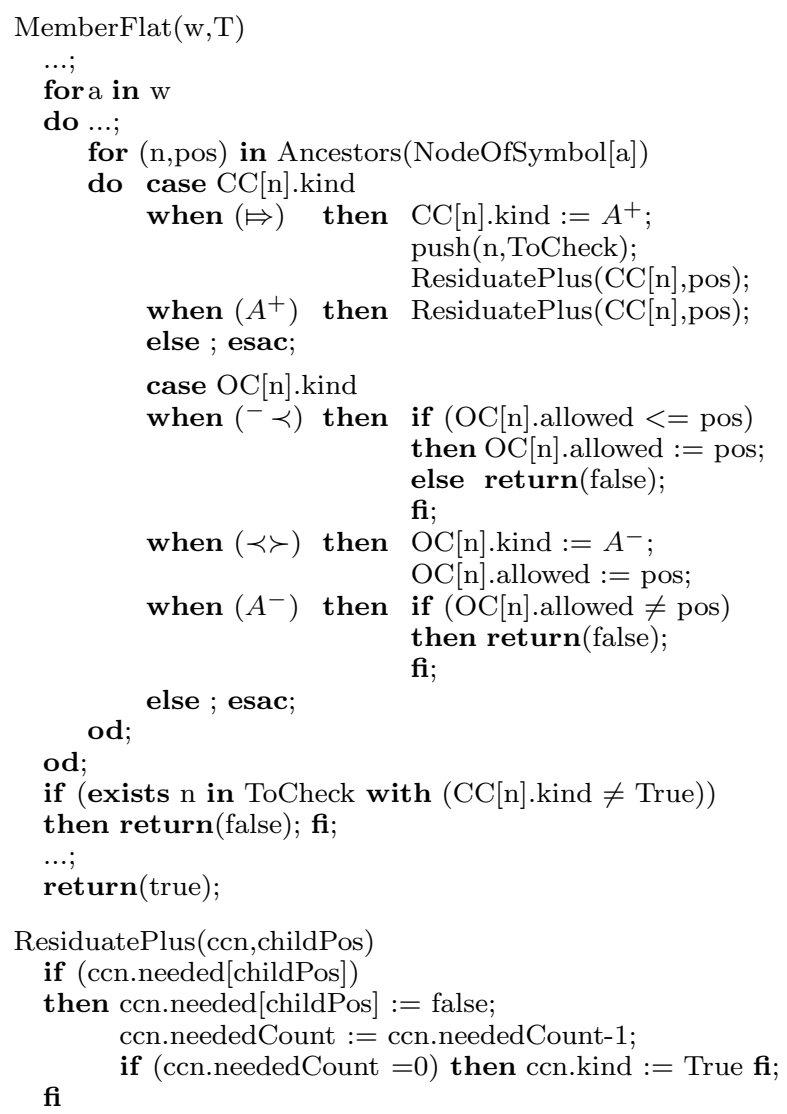

Figure 5: Checking with n-ary operators

Theorem 4.2 (soundness) MemberFlat $(w, T)$ yields true iff $w \in \llbracket T \rrbracket$.

Theorem 4.3 (complexity) MemberFlat $(w, T)$ runs in time $O(|T|+|w| *$ flatdepth $(T))$.

\section{THE LINEAR ALGORITHM}

We present here an orthogonal optimization, which makes the algorithm truly linear.

The base algorithm visits all the ancestors of NodeOfSymbol $[a]$ every time $a$ is found in $w$, which is redundant. Consider a node $n$ such that $A_{1}$ and $A_{2}$ are, respectively, the symbols in its left and right descendants. Whenever the constraint of $n$ has been residuated because a symbol of $A_{1}$ has been met, there is almost no reason to visit $n$ again because of a symbol from $A_{1}$ (and the same holds for $A_{2}$ ). There is only one exception: if the constraint is $A_{1} \prec A_{2}$, then, even after a symbol of $A_{1}$ has already been seen, a symbol from $A_{2}$ transforms the constraint into $A_{1}{ }^{-}$, and a further symbol from $A_{1}$ cannot be ignored, but will cause the algorithm to yield "false".

Formally, we have the following " $A_{2}$-stability" property. For any constraint $F$ with shape $A_{1}{ }^{+} \Leftrightarrow A_{2}{ }^{+}, A_{1}{ }^{+} \Leftrightarrow A_{2}{ }^{+}$, $A_{1} \prec \succ A_{2}$ or $A_{1} \prec A_{2}$, with $A_{1}$ disjoint from $A_{2}$, the following holds:

$$
a \in A_{2} \wedge F \stackrel{a w+}{\rightarrow} F^{\prime} \Rightarrow \forall a^{\prime} \in A_{2} F^{\prime} \stackrel{a^{\prime}}{\rightarrow} F^{\prime}
$$

The same property holds for $a \in A_{1}$ and $a^{\prime} \in A_{1}$ for all the constraints but $A_{1} \prec A_{2}$. Specifically, $A_{1}$-stability is only violated when a letter $b \in A_{2}$ is in $w$, as follows:

$$
a \in A_{1}, b \in A_{2}, a^{\prime} \in A_{1}:\left(A_{1} \prec A_{2}\right) \stackrel{a b}{\rightarrow}+\left(A_{1}{ }^{-}\right) \stackrel{a^{\prime}}{\rightarrow} \text { false }
$$

The linear algorithm exploits this observation as follows:

1. whenever an upward pointer Parent $[$ child], yielding (parent, direction), is followed, the same pointer is set to null, so that it will not be traversed again; however, the node child is stored in Deleted[parent,direction];

2. to deal with the $A_{1}-A_{2}-A_{1}$ case of the $A_{1} \prec A_{2}$ constraint, when a node parent marked with $\prec$ is reached from its right subtree, we use Deleted[parent,left] to recursively rebuild all the upward pointers in its left subtree; this is the only case when upward pointers are rebuilt.

Example 5.1 Consider the type $T=((a+\epsilon) \& b[1 . .5]) \cdot(c+$ $d^{+}$) (Figures 1, 6, and 7), and assume we read a word bbac. The first time we read $b$, when we visit the ancestor (2, right), we assign Parent $[5]=$ null and Deleted $[2$, right $]=5$, so that now 5 has no parent, but the fact that the right child of 2 was 5 is recorded (the pointer from 5 to 2 is "reversed"). When we then visit (1,left), we also assign Parent $[2]=$ null and Deleted $[1$, left $]=2$. The second time $b$ is read, the parent of 5 is found to be null, hence no action is taken, apart from counting $b$. When $a$ is read, its UnvisitedAncerstors list only contains (2,left), since the parent of 2 has been deleted. When node 2 is visited, we set Parent $[4]=$ null and Deleted $[2, l e f t]=4$. The situation is depicted in Figure 6 .

Now, the arrival of any letter $l$ from $\{a, b\}$ would only increment Count $[l]$, but no ancestor would be visited. When $c$ is read, both Parent [6] and Parent [3] are deleted, but, since $O C[1]=\prec$ and the direction is right, Reactivate(1, left) is invoked, which restores all pointers that are reachable 


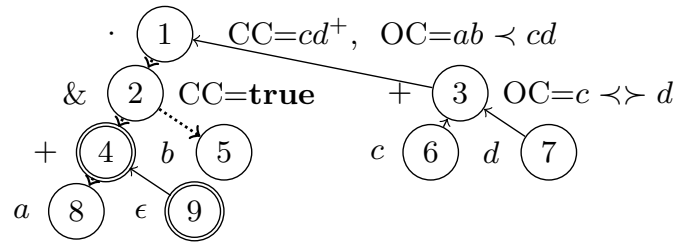

Figure 6: The tree for $T=((a+\epsilon) \& b[1 . .5]) \cdot\left(c+d^{+}\right)$ after word $b b a$ has been read; the dotted lines are those that are represented into the Deleted array.

from the left pointer of 1 ; the resulting tree is depicted in Figure 7.

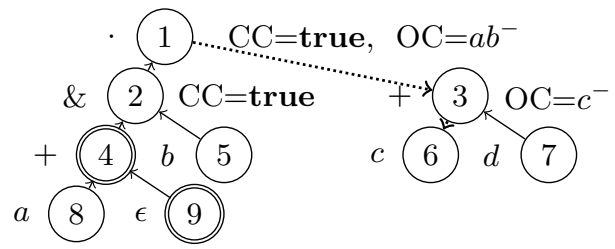

Figure 7: The tree for $T=((a+\epsilon) \& b[1 . .5]) \cdot\left(c+d^{+}\right)$ after word $b b a c$ has been read

The algorithm would now terminate with success. If a new $a$ or $b$ were read at this point, it would not be missed, but would cause the algorithm to stop with false.

No upward pointer can be deleted and rebuilt twice. Assume that an edge $e$ has been deleted and rebuilt; this only happens if $e$ is in the left subtree of a node $n$ with constraint $A_{1} \prec A_{2}$, and two symbols from $A_{1}$ and $A_{2}$ have been met, and hence $O C[n]$ is now $L^{-}$. If the same $e$ is deleted again, then the algorithm will return false as soon as the $n$ ancestor of $e$ is reached. Hence, any edge is traversed at most three times, to be deleted, rebuilt, and deleted for good. Similarly, the linear algorithm visits any internal node of the type at most three times, arriving twice from the left subtree and once from the right subtree. Hence, the algorithm has an $O(|T|)$ set-up cost, an $O(|w|)$ cost to access NodeOfSymbol $[a]$ for $|w|$ times, and a total residuation cost which is bound by $O(|T|)$, which gives a total of $O(|T|+|w|)$.

The initialization of the algorithm is identical to the nonoptimized version, apart from the construction of the empty Deleted [] array. The body of the algorithm only changes when pointers are cut, and in the management of the $(\prec$, right) case (Figure 8).

Theorem 5.2 (soundness) MemberLin $(w, T)$ yields true iff $w \in \llbracket T \rrbracket$.

Theorem 5.3 (complexity) MemberLin( $w, T)$ runs in time $O(|w|+|T|)$.

This optimization can be easily combined with flattening, obtaining a MemberFlatLin version, which would outperform MemberFlat in situations where we have long words with repeated characters, and would outperform MemberLin when types are "large", especially if the set-up phase can be shared by different runs of the algorithms, as discussed in the next section.

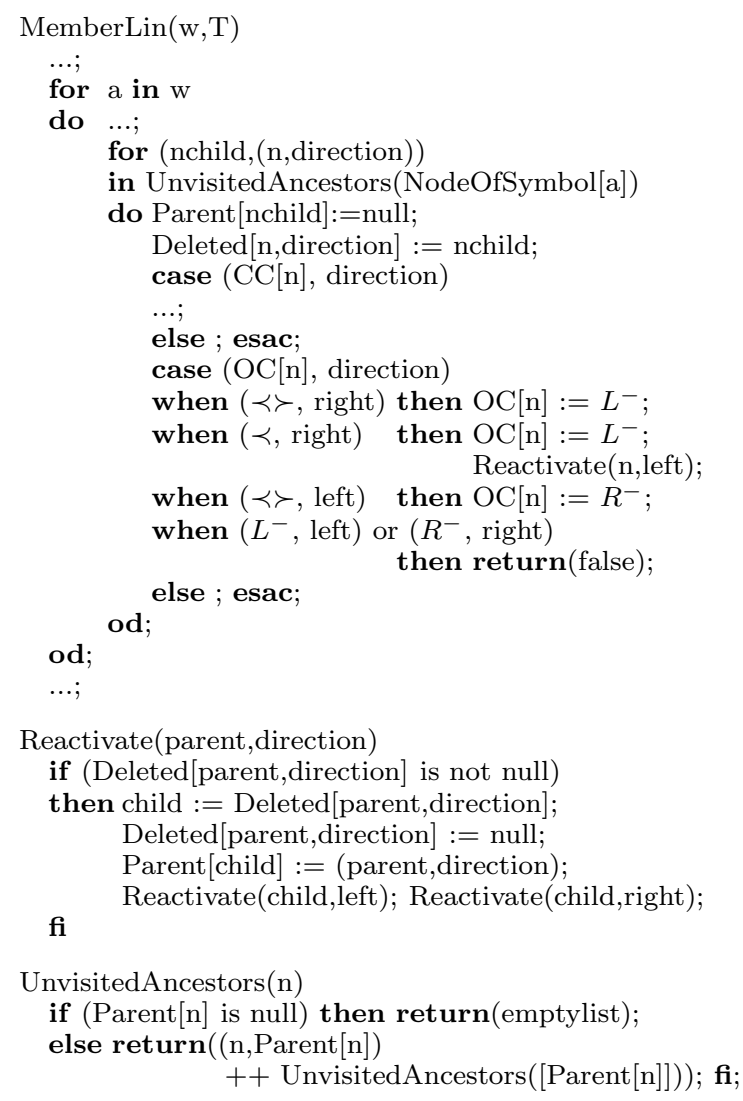

Figure 8: Version in $O(|w|+|T|)$

\section{MULTI-WORDS CHECKING}

We now study the multi-words membership problem, i.e., the case where one type $T$ is used to check $m$ words $w_{1}, \ldots, w_{m}$. The repeated application of MemberFlatLin gives us an upper bound of $m *(|T|+|w|)$, where $|w|$ is the average length of the words. This bound is not linear, in general, in the input size $|T|+(m *|w|)$. In the special case when $|T| \leq|w|$, then $m *(|T|+|w|)$ is smaller than $2 m *|w|$, hence the algorithm is indeed linear. In the general case, where $|T|$ may be much bigger than $|w|$, we get a better result if we avoid re-building the $T$ structure from scratch after each word is checked. To this aim, we build the Parent [], $C C$ [], OC [], etc., structures once, and we also build two copies CCSave [], OCSave[] of $C C[]$ and $O C[]$. We then run a version MultiFlatLin of the MemberFlatLin algorithm with an undo-enabling line

$$
\text { Updated[n,direction] := nchild; }
$$

added immediately after the line

$$
\text { Deleted[n,direction] := nchild; }
$$

After each word is checked, we apply the following code to the root of the type, to restore Parent [], $C C[], O C[]$, Updated [], Deleted [], and Count [] to their original state; we have first built a $S y m b o l[]$ array that associates each $a ?[m . . n]$ node with its symbol $a$.

OC[root]:= OCSave[root]; CC[root]:= CCSave[root];

RestoreChild(root,left); RestoreChild(root,right); where 
RestoreChild(parent,direction)

if (Updated[parent,direction] is not null)

then child $:=$ Updated[parent,direction];

Updated[parent,direction] := null;

Deleted[parent,direction] := null;

Parent[child] := (parent,direction);

OC $[$ child]:= OCSave[child];

CC [child $]:=$ CCSave[child];

if (Symbol[child] is not null)

then Count $[$ Symbol $[$ child $]]:=0$;

else RestoreChild(child,left);

RestoreChild(child,right);

fi;

fi

This restoring phase does not visit the whole $T$ but only the modified part, hence is in $O(\min (|T|,|w| *$ flatdepth $(T)))$, hence, after a set-up phase with cost $O(|T|)$, the cost of checking each word is in $O(\min (|w|+|T|,|w| *$ flatdepth $(T)))$.

In the "easy case", when $|T|$ is smaller than $|w|$, each word is checked in $O(|w|)$, including the $O(|T|)$ time needed to setup and restore the $T$ structure, which gives the same linear complexity $O(m *|w|)$ as if $T$ were rebuilt from scratch. In the "hard case", when $|T|$ is not bound by $|w|$, we at least know that this algorithms checks each word, and restores the structures, in time $O\left(\left|w_{i}\right| *\right.$ flatdepth $\left.(T)\right)$, giving a total complexity of $O(|T|+m *|w| *$ flatdepth $(T))$. If we assume a constant upper-bound $k$ for flatdepth $(T)$, the complexity is in $O(|T|+m *|w| * k)$, hence is still linear in the input size. Without the "restoring" optimization, the total cost would be $O(m *(|T|+|w|))$, which is much worse, since, in practice, we cannot reasonably assume an upper bound on either $m$ or $|T|$.

\section{MEMBERSHIP FOR XSD SCHEMAS}

We are now ready to extend our techniques from words to trees. For the purpose of this discussion, we focus on XML trees where every node is an element node, hence on forests generated by the following grammar: $x:=\epsilon \mid\langle a\rangle x\langle/ a\rangle x$.

Following a long tradition, (see [9], for example), we model an XSD schema as an extended DTD, that is, as a quintuple $(\Sigma, \Delta, \tau, \mu, \rho)$, where $\Sigma$ is a set of labels, $\Delta$ is a set of type-names, $\tau$ is a function mapping each type-name to a content-model, which is a type expressed on the alphabet $\Delta, \mu$ is a function from $\Delta$ to $\Sigma$, and $\rho \in \Delta$ is the root type-name. Although $\mu$ is not injective in general, the $\mathrm{El}$ ement Declarations Consistent (EDC) constraint specifies that $\mu$ must be injective when restricted to a specific content model (see [16]). As a consequence, it is possible to check membership of an XML tree $x$ into an XSD schema as follows. Membership checking happens in the context of a specific type-name $\beta$, which is initially the root type-name of the schema, hence of a specific content-model $T=\tau(\beta)$. To check whether $\left\langle a_{1}\right\rangle x_{1}\left\langle/ a_{1}\right\rangle \ldots\left\langle a_{n}\right\rangle x_{n}\left\langle/ a_{n}\right\rangle$ satisfies $T$, we retrieve the content model $T_{i}=\tau\left(\mu_{\beta}^{-1}\left(a_{i}\right)\right)$ of each subelement, check that each $x_{i}$ matches $T_{i}$, and check that the sequence $w=\mu_{\beta}^{-1}\left(a_{1}\right) \ldots \mu_{\beta}^{-1}\left(a_{n}\right)$ matches $T$. Here, $\mu_{\beta}^{-1}\left(_{-}\right)$ is the inverse of $\mu$ restricted to the type-names appearing in the content model of $\beta$; this inverse function is well-defined thanks to the EDC constraint.

We assume here that each content model is expressed in our type language and satisfies the conflict-freedom constraint. The cost of verifying whether $x$ satisfies $(\tau, \mu, \rho)$ depends on the cost of checking whether a word belongs to a content model $\tau(\alpha)$, as follows. We assume that the XSD schema contains $|J|$ content models $\left\{\tau\left(\alpha_{j}\right)\right\}^{j \in J}$, each of size $\left|\tau\left(\alpha_{j}\right)\right|$, that $x$ contains (immediately or recursively) $|I|$ elements $\left\{e_{i}\right\}^{i \in I}$, and that $w_{i}$ is the sequence of the labels of the children of $e_{i}$. We assume that MultiFlatLin is used for word-membership. We have a set-up phase with cost $O\left(\sum_{j \in J}\left|\tau\left(\alpha_{j}\right)\right|\right)=O(|\tau|)$. We have a checking phase with cost $O\left(\min \left(\left|\tau\left(\alpha_{i}\right)\right|+\left|w_{i}\right|,\left|w_{i}\right| *\right.\right.$ flatdepth $\left.\left.\left(\tau\left(\alpha_{i}\right)\right)\right)\right)$ for each $w_{i} \in \llbracket \tau\left(\alpha_{i}\right) \rrbracket$ test. $^{2}$ If the size of each $w_{i}$ dominates the size of $\tau\left(\alpha_{i}\right)$, then the total cost is linear, by

$$
\begin{aligned}
& \sum_{i \in I} \min \left(\left|\tau\left(\alpha_{i}\right)\right|+\left|w_{i}\right|,\left|w_{i}\right| * \text { flatdepth }\left(\tau\left(\alpha_{i}\right)\right)\right) \\
& \leq \sum_{i \in I}\left(\left|\tau\left(\alpha_{i}\right)\right|+\left|w_{i}\right|\right) \\
& \leq 2 \sum_{i \in I}\left|w_{i}\right| \leq 2|x|
\end{aligned}
$$

Here we exploit the optimization described in Section 5. Observe that it is not true, in general, that $\sum_{i \in I}\left|\tau\left(\alpha_{i}\right)\right| \leq|\tau|$ since the set $I$ enumerates the elements inside $x$, not the components of $\tau$.

This linear approximation does not hold when $\tau\left(\alpha_{i}\right)$ may be bigger than $w_{i}$, as happens in cases where complex content models are used to check documents where each element has a small number of children. In this case, which is quite common, we still have a quasi-linear complexity, by:

$$
\begin{aligned}
& \sum_{i \in I} \min \left(\left|\tau\left(\alpha_{i}\right)\right|+\left|w_{i}\right|,\left|w_{i}\right| * \text { flatdepth }\left(\tau\left(\alpha_{i}\right)\right)\right) \\
& \leq \sum_{i \in I}\left(\left|w_{i}\right| * \text { flatdepth }\left(\tau\left(\alpha_{i}\right)\right)\right) \\
& \leq\left(\sum_{i \in I}\left|w_{i}\right|\right) * \max \left(\text { flatdepth }\left(\tau\left(\alpha_{i}\right)\right)\right) \\
& \leq|x| * \max \left(\text { flatdepth }\left(\tau\left(\alpha_{i}\right)\right)\right)
\end{aligned}
$$

This upper bound is linear if we assume a constant upper bound $k$ for flatdepth $\left(\tau\left(\alpha_{i}\right)\right)$. Here we exploit the combined optimizations described in Section 4 and Section 6 .

Since DTDs can be modeled as a special case of EDTDs, this quasi-linearity result holds for DTDs as well.

\section{EXPERIMENTAL EVALUATION}

To validate the usefulness of our approach and its theoretical properties, we built a prototype implementation of our linear algorithm (Xelf), and briefly analyzed its scalability properties when used on XML trees. To better understand the behaviour of our approach, we also compared our implementation with a validating SAX parser and a nonvalidating SAX parser.

We analyze the behaviour of Xelf on XML documents of increasing size, so to expose its scalability properties.

\subsection{Experimental Setup}

Our algorithm has been implemented in Java 1.5 and all experiments were performed on a $2.16 \mathrm{Ghz}$ Intel Core 2 Duo machine (1 GB main memory) running Mac OSX 10.5.2. To avoid issues related to independent system activities, we ran each experiments five times, discarded both the highest (worst) and the lowest (best) processing times, and reported the average processing time of the remaining runs.

We compared our algorithm with the standard SAX parsers of Java 1.5 (based on Xerces [1]).

\footnotetext{
${ }^{2}$ Although XSD-checking uses top-down recursion, its total run-time can be still evaluated by just adding the time needed to verify that the $w_{i}$ label sequence of each element, at any depth level in the document, matches the element content model
} 


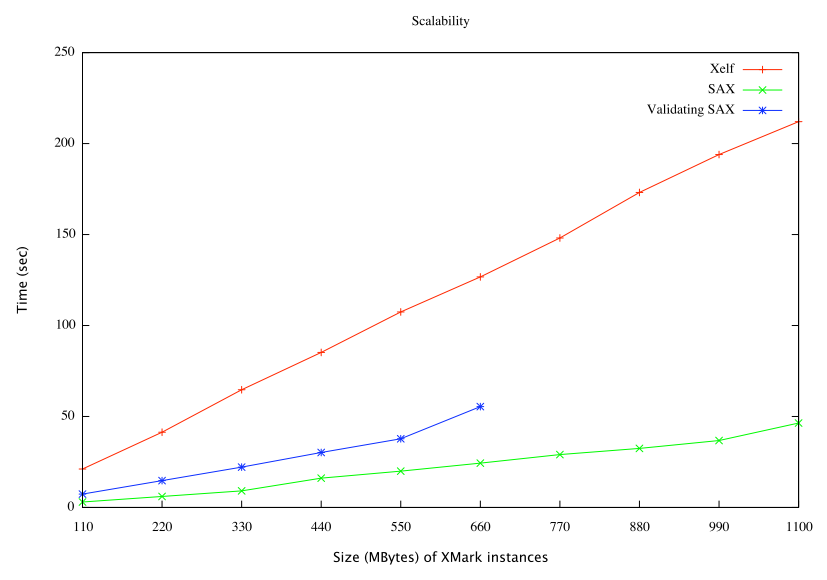

Figure 9: Scalability of Xelf.

We evaluated our system on a dataset containing 10 instances of XMark [15], ranging from 110 MBs to 1.09 GBs.

\subsection{Experiments}

The experimental results we obtained are shown in Figure 9. First of all, these results show a linear behavior, hence confirming our complexity analysis. Furthermore, as illustrated by the diagram, our approach is extremely scalable, while the validating SAX parser was unable to complete the validation process on documents of size larger than 680 MBytes, due to memory consumption; this suggests that our algorithm has a limited memory footprint and that it can be profitably used for online validation.

This suggestion has been confirmed by further experiments, where we measured the memory used by our approach during the validation of our dataset: in these experiments our algorithm used no more than 301 KBytes during validation, even on very big documents.

We do not precisely know why the validating SAX parser fails in validating large XMark documents: we believe that the deep nesting of XMark documents may have caused the problem, but we have no real evidences.

As we expected, our prototype implementation is slower than the validating SAX parser; however, our implementation is just a proof-of-concept, while Xerces is a longstanding and highly optimized parser.

\section{CONCLUSIONS}

Membership checking is NP-hard for REs with interleaving. We have presented here a subclass of these REs which admits a simple polynomial membership algorithm. The algorithm is based on the transformation of the RE into a set of constraints, and on the parallel incremental residuation of these constraints. We have discussed the practical relevance of this class of extended REs, and have presented some optimizations that make our algorithm linear in the size of $|T|+|w|$. Apart from the practical motivations, we believe that it is important to understand how far the expressive power of REs can be extended with "hard" operators such as interleaving and counting before making membership NP-hard. Our algorithm is not linear when used to check $m$ words $\left\{w_{i}\right\}_{i \in 1 . . m}$ against one type $T$, since $T$ appears once in the input, but it is visited $m$ times by the algorithm. We have presented an optimization that makes the algorithm almost linear for repeated checking, that is, makes it linear in $|T|+\left(\sum_{i \in 1 . . m}\left|w_{i}\right|\right) *$ flatdepth $(T)$, and flatdepth $(T)$ is very small in practice. Repeated checking is at the heart of XML membership checking with respect to DTDs and XSD schemas, hence the same quasi-linear complexity is preserved when we use our approach for XML membership checking. Finally, we experimentally validated the scalability properties of our approach.

\section{REFERENCES}

[1] http://xerces.apache.org/.

[2] D. Barbosa, G. Leighton, and A. Smith. Efficient incremental validation of XML documents after composite updates. In XSym, volume 4156 of $L N C S$, pages 107-121. Springer, 2006.

[3] D. Barbosa, A. O. Mendelzon, L. Libkin, L. Mignet, and M. Arenas. Efficient incremental validation of XML documents. In $I C D E$, pages 671-682. IEEE Computer Society, 2004.

[4] G. J. Bex, F. Neven, and J. V. den Bussche. DTDs versus XML schema: A practical study. In $W e b D B$, pages 79-84, 2004.

[5] G. J. Bex, F. Neven, T. Schwentick, and K. Tuyls. Inference of concise DTDs from XML data. In $V L D B$, pages 115-126, 2006.

[6] G. J. Bex, F. Neven, and S. Vansummeren. Inferring XML schema definitions from XML data. In $V L D B$, pages 998-1009, 2007.

[7] J. Brzozowski. Derivates of regular expression. Journal of the ACM, 11:481-494, 1964.

[8] B. Choi. What are real DTDs like? In $W e b D B$, pages 43-48, 2002.

[9] W. Gelade, W. Martens, and F. Neven. Optimizing schema languages for XML: Numerical constraints and interleaving. In ICDT, 2007.

[10] G. Ghelli, D. Colazzo, and C. Sartiani. Efficient inclusion for a class of XML types with interleaving and counting. In $D B P L, 2007$.

[11] H. Hosoya and B. C. Pierce. XDuce: A statically typed XML processing language. ACM Transactions on Internet Technology, 3(2):117-148, May 2003.

[12] J.E. Hopcroft and J.D. Ullman. Introduction to Automata Theory, Languages and Computation. Addison-Wesley, 1979.

[13] A. J. Mayer and L. J. Stockmeyer. Word problems this time with interleaving. Inf. Comput., 115(2):293-311, 1994.

[14] M. Montazerian, P. T. Wood, and S. R. Mousavi. XPath query satisfiability is in PTIME for real-world DTDs. In XSym, volume 4704 of $L N C S$, pages 17-30. Springer, 2007.

[15] A. Schmidt, F. Waas, M. Kersten, D. Florescu, I. Manolescu, M. J. Carey, and R. Busse. The XML Benchmark Project. Technical report, Centrum voor Wiskunde en Informatica, April 2001.

[16] H. S. Thompson, D. Beech, M. Maloney, and N. Mendelsohn. XML Schema Part 1: Structures Second Edition. Technical report, World Wide Web Consortium, Oct 2004. W3C Recommendation.

[17] P. T. Wood. Containment for xpath fragments under DTD constraints. In ICDT, pages 300-314, 2003. 